\title{
Influence of Replacement Ratio of Recycled Concrete Aggregates on Flexural Behavior of Concrete Beams
}

\author{
Omar Aljidda ${ }^{1}$, Wael Alnahhal ${ }^{2}$
}

\begin{abstract}
This study investigates the effect of using recycled concrete aggregates (RCA) on the flexural behavior and ultimate capacity of reinforced concrete beams experimentally. A total of four reinforced concrete (RC) beam specimens were flexural tested until failure. The parameter investigated included the RCA replacement ratio $(0 \%, 25 \%, 50 \%$, and $100 \%)$. The testing results of the specimens were compared to control beam specimens made with natural coarse aggregates (NCA). In addition, the experimental results of beams with RCA were compared to the result of the beam without RCA. Test Results showed there was no remarkable effect of using RCA on the flexural strength of the tested beams.
\end{abstract}

Keywords- Recycled Concrete Aggregates, Flexural Behavior, Concrete Beams, Sustainable Materials.

\section{Introduction}

Concrete is the major construction material in Gulf region and in most countries of the world. However, Qatar suffers from the lack of the natural resources needed for concrete production. It is well known that $60 \%$ to $75 \%$ of the concrete volume is made up of aggregates. Most of the used aggregates in Qatar are imported from other countries, which will add extra transportation cost to the total cost of the aggregates. For example, the quantities of imported aggregates in Qatar have boosted from 9.5 million tons in 2006 to 21.5 million tons in 2008. About the 12 million tons of the solid waste generated in Qatar every year is from construction and demolition waste (C\&D), it is imperative to investigate the possibility of reusing $C \& D$ as alternative aggregates to the natural coarse aggregates (NCA). Approximately 20,000 tons of concrete debris is discarded every day, and half of that quantity is converted to RCA [1]. The use of recycled concrete aggregates (RCA) in concrete applications has major economic and environmental benefits. RCA can partially replace the relatively expensive imported NCA, thus saving on the total cost of the concrete projects. Recycling C\&D waste will also have a significant environmental impact due to the reduction in demand for the natural aggregates. Moreover, using waste materials would partially solve a significant environmental concern about landfilling the large quantities of $\mathrm{C} \& \mathrm{D}$ waste produced every year. There has been always a general belief about the unwanted influence of using RCA in concrete production. That is because of the presence of two interfacial transition zones (ITZ) in the RCA concrete (ITZ) between NCA and residual mortar in RCA and ITZ between the residual mortar and the fresh mortar compared with only one ITZ in the NCA concrete.

\footnotetext{
Graduate Student, Department of Civil and Architectural Engineering, Qatar University, Qatar.

2 Assistant Professor, Department of Civil and Architectural Engineering, Qatar University, Qatar. Corresponding Author.
}

However, number of publications have contradicted this idea [2-4]. Several researchers have examined the performance of RCA in structural concrete [5-8]. It was stated that RCA has inferior water absorption and porosity characteristics in comparison to NCA [1]. Therefore, a proper mix design is necessary to obtain the required quality of concrete made of RCA [9]. In general, the quality of RCA is governed by the quality of the original concrete it had been recycled from. Silva et al. [10] showed that classification of RCA based on their quality can be used to produce concrete with an expectable performance. McNeil and Kang [11] reported that using RCA resulted in a decrease in the compressive strength, the modulus of rupture and the modulus of elasticity of RCA concrete compared to normal concrete. In addition, several publications reported the effect of different RCA's surface pretreatment methods on fresh and hardened properties of concrete mixtures made with surface treated RCA [12-14]. Yehia et al. [15] studied the properties of concrete with $100 \%$ content of RCA in terms of strength and durability. According to the authors, the strength and quality of RCA depend on the condition of exposure and loading condition of demolished structures.

A few number of studies investigated experimentally the flexural properties of RC beams made with RCA. Choi and Yun [16] studied the flexural behavior and long-term deflection of beams made with RCA under sustained loads for a period of 380 days. According to their study, the maximum flexural strength of beams made with NCA was $20 \%$ higher than that of the beams with RCA. Similar crack patterns were observed regardless of the aggregates type. However, more cracks were present in beams made with RCA. The ratios of long-term to instantaneous deflection for beams made with RCA were smaller than the ratio for the beam made with NCA. In addition, Arezoumandi et al. [17] reported that deflection corresponding to the ultimate flexural strength of a beam made with RCA is about 13\% higher than the comparable beam made with NCA. Knaack and Kurama [18] tested beams with both $50 \%$ and $100 \%$ RCA replacement level. They reported an increase in the deflections as the amount of RCA is increased. Similar results were reported by Song et al. [19] However, they also reported that the addition of RCA into concrete has little effect on the ultimate moment capacity and the flexural crack width of the tested specimens.

\section{The Experimental Program}

The experimental program comprises two parts: the first part aims at investigating the mechanical properties of concrete made with RCA in terms of compressive and flexural strengths. The second part aims at investigating the flexural behavior of beams content RCA under four-point loading test. 
Proc. of the Sixth International Conference on Advances in Civil, Structural and Environmental Engineering - ACSEE 2017. Copyright $(\odot$ Institute of Research Engineers and Doctors. All rights reserved.

ISBN: 978-1-63248-139-9 doi: 10.15224/ 978-1-63248-139-9-33

\section{A. Concrete Mixture Proportions}

Washed sand was used as fine aggregates in all concrete mixes. The cement was an Ordinary Portland Cement (OPC) was used in the mix. The two coarse aggregates in this research were NCA (Gabbro) and RCA with a maximum size of $20 \mathrm{~mm}$. The water/cement ratio of the mix was 0.45 . The target compressive strength of concrete was $35 \mathrm{MPa}$. four different replacement ratios of RCA namely $0 \%, 25 \%, 50 \%$ and $100 \%$ by volume were added. As the RCA is characterized by its higher water absorption due to the attached old mortar compared with NCA, the amount of free water in concrete mixture plays a significant rule in the development of the hardened properties for resulting concrete. For this reason, RCA and NCA were washed and immersed in water for 24 hours before mixing, and then the surface moisture was dried with a moistened cloth, as shown in Figure1.This is to ensure that both aggregates were in a saturated surface dry condition at the time of concrete mixing. Water absorbed by saturated aggregates was not included in calculating water to cement content. This is to ensure that the remaining amount of water is approximately the same and is enough to ensure the hydration of the cement particles in the concrete mix. The direct volume replacement (DVR) mix design method was adopted to calculate the concrete mixture proportions.

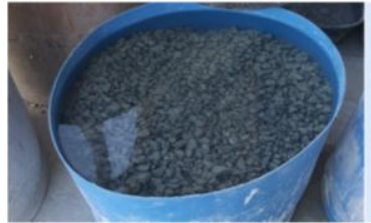

a.) Moisturizing of NCA

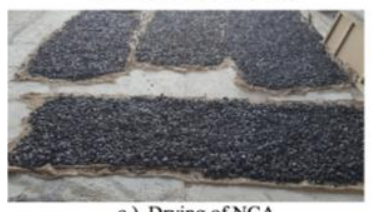

c.) Drying of NCA

Figure 1: Moisturizing and Drying of Aggregates.

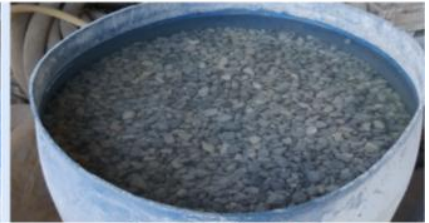

b.) Moisturizing of RCA

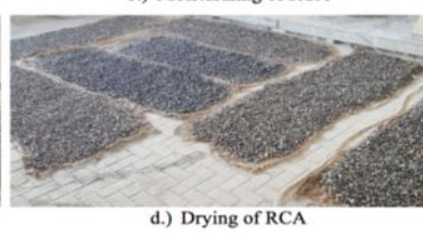

\section{B. Flexural and Compression Strength Test}

The flexural and compression tests were conducted in accordance with ASTM C78-10 and ASTM C39-16. For the flexural tensile strength test, prisms with a size of $100 \times 100 \times$ $500 \mathrm{~mm}$ were tested, and the average of three specimens for each mix was selected. For the compressive test, three cylinders from each mix with a dimension of $150 \times 300 \mathrm{~mm}$ were tested then the average compressive strength was selected. All specimens were cured for 28 days and tested thereafter.

\section{Large-Scale Beams Flexural Test}

To study the flexural behavior of beams content RCA, test was carried on large-scale beams with a size of $150 \times 250 \times$ $2550 \mathrm{~mm}$ that were loaded until failure under four-point loads as shown in Figure 2. steel bars were used for the reinforcement in the construction of beams, the bars of diameter $8 \mathrm{~mm}$ were used for all transverse steel reinforcement (stirrups) and used as compression reinforcement (top steel) for all the beams, while the $16 \mathrm{~mm}$ bars were used for the main flexural steel reinforcement.

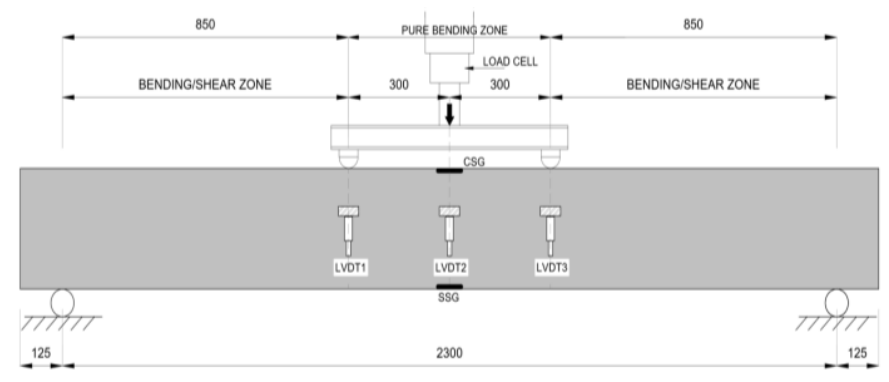

Figure 2: Large-Scale Beam Layout (Dimensions in $\mathrm{mm}$ )

\section{Tests Results and Discussion}

\section{A. Compressive Test Results}

Figure 3 illustrates the average compressive strength of three cylinders tested at 28 days with different replacement ratios of RCA $(0 \%, 25 \%, 50 \%$, and $100 \%)$. It can be observed that there is a minor effect on compressive strength by the replacement of RCA even up to a $100 \%$, and the general observed trend is similar to the compressive strength of concrete with NCA. This is mainly due to use the direct volume replacement (DVR) concrete mix design method and using RCA in saturated surface dry condition seemed to impose the largest positive effect on the concrete compressive strength due to water presence in RCA. That is because the compressive strength of concrete made with RCA depends on mix proportions, the moisture of RCA, and the method of treatment of RCA.

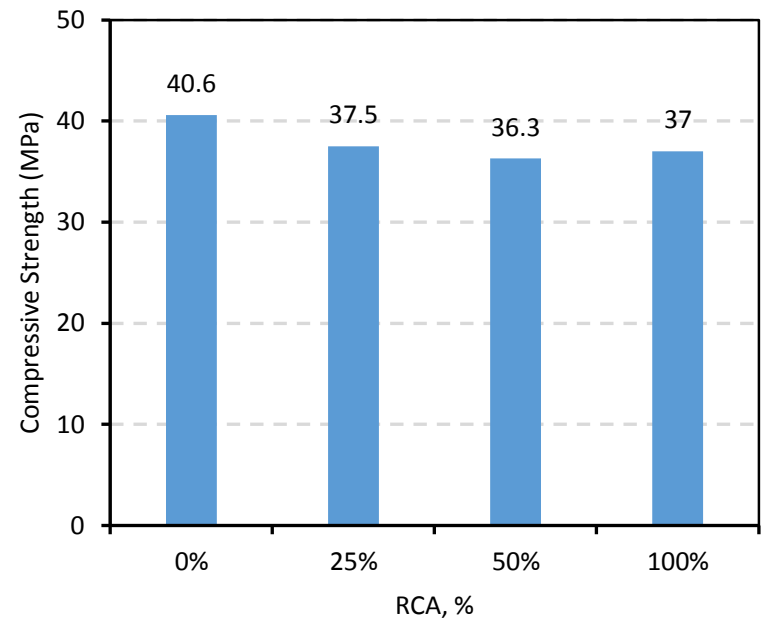

Figure 3: Compressive Strength Test Results for Different RCA Replacement Ratios

\section{B. Flexural Test Results}

Figure 4 presents the average flexural tensile strength of all concrete mixtures with different replacement ratios of RCA. 
The testing results showed that the addition of RCA at different replacement percentage ratios of $25 \%, 50 \%$, and $100 \%$ has a slight effect on the flexural tensile strength of the concrete mixtures. It can be observed that there is no clear correlation between the two parameters. It can be observed that replacing NCA by RCA in concrete resulted in a slight increase of $2.89 \%$ and $1.35 \%$ the flexural strength when the replacement ratios of the RCA increased to $25 \%$ and $50 \%$ respectively compared to control prism and slight decrease of $1.83 \%$ when the replacement ratios of the RCA increased to $100 \%$ compared to control prism.

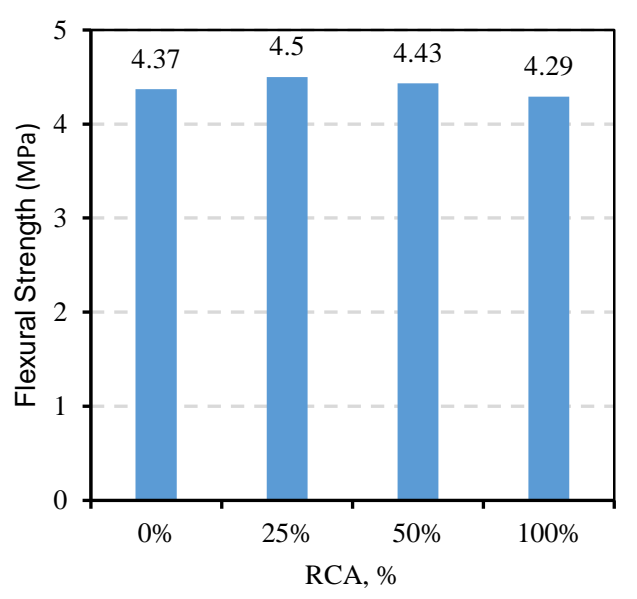

Figure 4: Flexural Tensile Strength Test Results for Different RCA Replacement Ratios

\section{Flexural Test Results for Large-Scale Beams}

All beams were loaded gradually and uniformly until reaching the failure load. The development of cracks in each RC beams was tracked and noted constantly. The cracking, yielding and ultimate loads and moments, maximum midspan deflection for each beam. As anticipated, the influence of using RCA on the flexural strength of the tested beams was relatively small. This finding is in a good agreement with the concrete compressive strength testing results presented in section A. As shown in Figure 5 (a), as the RCA replacement ratio increased, beams presented a slightly smaller ultimate flexural strength and deflection at failure. This is primarily due to the presence of the attached old mortar. The decrease in the maximum deflection at failure was $8.57 \%, 11.43 \%$, and $14.29 \%$ when the replacement ratio of the RCA increased to $25 \%, 50 \%$, and $100 \%$ respectively. In addition, the replacement of NCA with RCA resulted in a slight decrease in the ultimate flexural capacity of $8.82 \%, 6.95 \%$ and $5.18 \%$ for beams with $25 \%, 50 \%$, and $100 \%$ RCA replacement ratios respectively, compared to the control beam made with NCA. Figure 5 (b) shows the load-concrete strain relationships for beams containing no BMF with different replacement ratios of RCA. It was observed that there is no correlation between the concrete strain and the replacement ratio of RCA, since increasing the replacement ratio of RCA did not influence the concrete compressive strain.

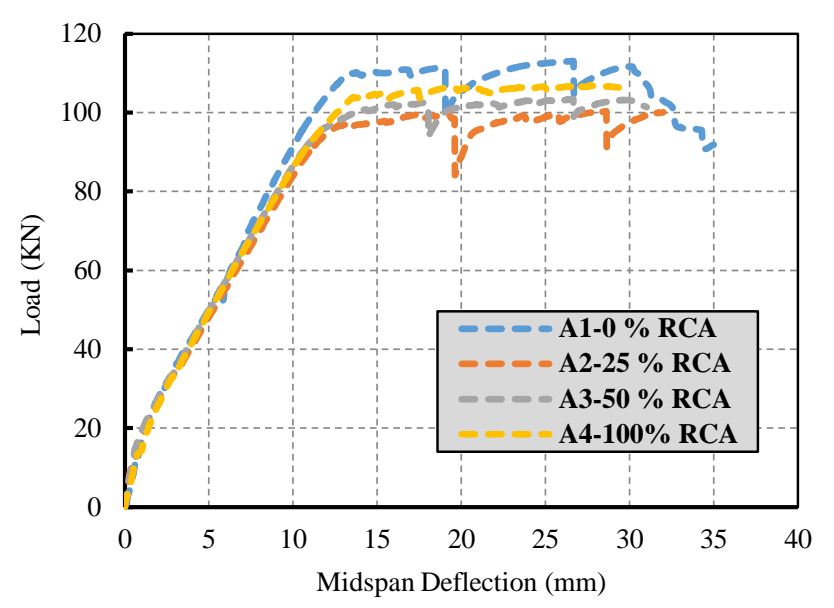

(a)

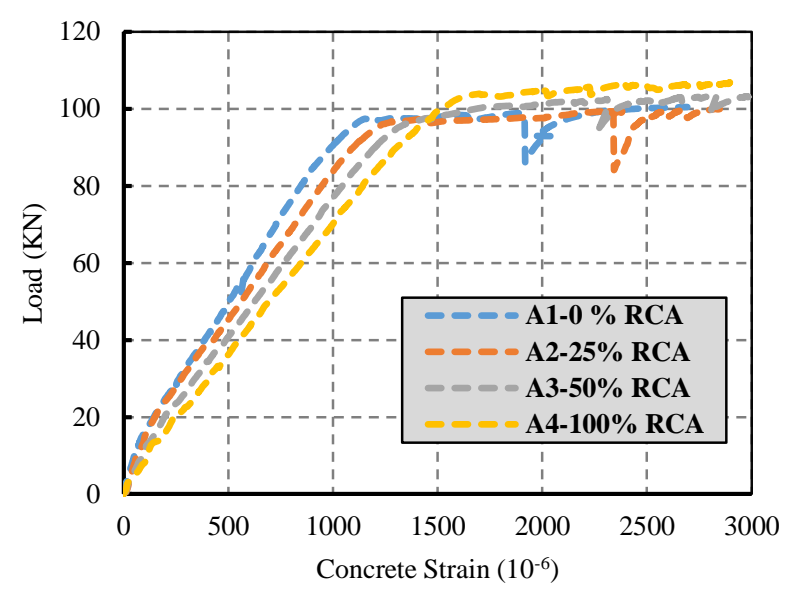

(b)

Figure 5: Comparison of Test Results for Beams with Different Replacement Ratios of RCA

\section{Conclusion}

In conclusion, this study has investigated the mechanical properties and flexural behavior of RC beams with different replacement ratios of RCA. The following conclusions can be drawn based on the findings of the experimental investigation:

- The addition of RCA into concrete has little-to-no effect on both compressive and flexural tensile strengths of the concrete mix. This is mainly due to the use of the absolute volume concrete mixing method with saturated surface dry RCA.

- The influence of using RCA in concrete mixtures on the flexural strength of the tested beams was relatively small even at full replacement.

- The addition of RCA into concrete has little effect on the flexural tensile strength of the concrete mix, This is because the negative effect related to the weakness of the old interfacial transition zone in RCA. 


\section{Acknowledgment}

The authors show their gratitude to Qatar University for their financial support through the internal research grant QUUS-CENG-SPR14/15-21. The authors are grateful to the Construction Materials Co L.L.C. (Qatar Quarry Company) for providing the RCA.

\section{References}

[1] M. Al-Ansary and S. R. Iyengar, "Physiochemical characterization of coarse aggregates in Qatar for construction industry,” Int. J. Sustain. Built Environ., vol. 2, no. 1, pp. 27-40, 2013.

[2] M. L. Berndt, "Properties of sustainable concrete containing fly ash , slag and recycled concrete aggregate," Constr. Build. Mater., vol. 23, no. 7, pp. 2606-2613, 2009.

[3] S. W. Tabsh and A. S. Abdelfatah, "Influence of recycled concrete aggregates on strength properties of concrete," Constr. Build. Mater., vol. 23, no. 2, pp. 1163-1167, 2009.

[4] O. Kayali, M. N. Haque, and J. M. Khatib, "Sustainability and Emerging Concrete Materials and Their Relevance to the Middle East," The Open Construction and Building Technology Journal, vol. 2, no. 1, pp. 103$110,2008$.

[5] M. C. Roa, S. K. Bhattacharyya, and S.V. Barai, "Influence of field recycled coarse aggregate on properties of concrete," Materials and Structures, vol. 44, no. 1, pp. 205-220, 2011.

[6] v. Corinaldesi, "Mechanical and elastic behaviour of concretes made of recycled-concrete coarse aggregates," Constr. Build. Mater., vol. 24, no. 9, pp. 1616-1620, 2010.

[7] Y.Lin, Y. Tyan, T. Chang, and C. Chang, "An assessment of optimal mixture for concrete made with recycled concrete aggregates," Cement and concrete research, vol. 34, pp. 1373-1380, 2004.

[8] L. Evangelista and J. De Brito, "Durability performance of concrete made with fine recycled concrete aggregates," Cem. Concr. Compos., vol. 32, no. 1, pp. 9-14, 2010.

[9] S.Kou, C. Poon, and F. Agrela, "Composites Comparisons of natural and recycled aggregate concretes prepared with the addition of different mineral admixtures," Cem. Concr. Compos., vol. 33, no. 8, pp. 788-795, 2011.

[10] R. V. Silva, J. De Brito, and R. K. Dhir, "Properties and composition of recycled aggregates from construction and demolition waste suitable for concrete production," Constr. Build. Mater., vol. 65, pp. 201-217, 2014.

[11] K. McNeil and T. H. K. Kang, "Recycled Concrete Aggregates: A Review,” Int. J. Concr. Struct. Mater., vol. 7, no. 1, pp. 61-69, 2013.

[12] Y.-C. Liang, Z.-M. Ye, F. Vernerey, and Y. Xi, "Development of Processing Methods to Improve Strength of Concrete with 100\% Recycled Coarse Aggregate," J. Mater. Civ. Eng., vol. 27, no. 5, p. 130801045339002, 2013.

[13] R. Purushothaman, R. R. Amirthavalli, and L. Karan, "Influence of Treatment Methods on the Strength and Performance Characteristics of Recycled Aggregate Concrete," J. Mater. Civ. Eng., vol. 27, no. 5, 2000.

[14] E. Güneyisi, M. Gesoğlu, Z. Algın, and H. Yazıcı, "Effect of surface treatment methods on the properties of self-compacting concrete with recycled aggregates," Constr. Build. Mater., vol. 64, pp. 172-183, 2014.

[15] S. Yehia, K. Helal, A. Abusharkh, A. Zaher, and H. Istaitiyeh, "Strength and Durability Evaluation of Recycled Aggregate Concrete," Int. J. Concr. Struct. Mater., vol. 9, no. 2, pp. 219-239, 2015.

[16] W.-C. Choi and H.-D. Yun, "Long-term deflection and flexural behavior of reinforced concrete beams with recycled aggregate," Mater. Des., vol. 51, pp. 742-750, 2013.

[17] M. Arezoumandi, A. Smith, J. S. Volz, and K. H. Khayat, "An experimental study on flexural strength of reinforced concrete beams with $100 \%$ recycled concrete aggregate," Eng. Struct., vol. 88, pp. 154$162,2015$.
[18] A. M. Knaack and Y. C. Kurama, "Behavior of Reinforced Concrete Beams with Recycled Concrete Coarse Aggregates," Journal of Structural Engineering, vol. 141, no. 3, 2015.

[19] S. Song, K. Choi, Y. You, K. Kim, and H. Yun, "Flexural Behavior of Reinforced Recycled Aggregate Concrete Beams," Journal of the Korea Concrete Institute, vol. 21, no. 4, pp. 431-439, 2009. 